\title{
An Improved Method for Mapping Tidal Flats Based on Remote Sensing Waterlines: A Case Study in the Bohai Rim, China
}

\author{
Xiangyang Liu, Zhiqiang Gao, Jicai Ning, Xiang Yu, and Yuanyuan Zhang
}

\begin{abstract}
Tidal flats form boundaries between marine and terrestrial environments, and reported rapid decreases in their extent require improved measures of their status. Current methods for delineating tidal flats have low accuracy because of the limited precision of available imagery, and they cannot identify zones within tidal flats. Three zones within tidal flats are ecologically distinct due to their varied inundation. These include the supratidal, intertidal, and subtidal zones. In this study, we present a more precise method for mapping tidal flats. We used Chinese HJ-1A/B satellite images with a semiautomatic extraction method to generate a sufficient number of waterline estimates taken at various tide heights to delineate the full extent of tidal flats. Then, the full set of waterline estimates is analyzed with the Digital Shoreline Analysis System (DSAS) using Jenks Natural Breaks to identify three zones within tidal flats. We demonstrated the utility of this approach by mapping tidal flats of the Bohai Rim, China. In 2014, the estimate of available tidal flats in the region was $3093 \mathrm{~km}^{2}$, among which the area of supratidal, intertidal, and subtidal zones accounted for $\mathbf{5 . 0 \%}, \mathbf{8 4 . 3 \%}$, and $\mathbf{1 0 . 7 \%}$, respectively. A confusion matrix for accuracy assessment revealed a $93.8 \%$ overall accuracy for the resulting tidal flat map. Our method enables tidal flats to be mapped and monitored precisely and comprehensively, providing baseline data on the extent of this disappearing ecosystem that can be used to inform coastal planning and sustainable development.
\end{abstract}

Index Terms-Digital Shoreline Analysis System (DSAS), HJ image, Jenks Natural Breaks, tidal flat, waterline, zones.

\section{INTRODUCTION}

$\mathbf{T}$ IDAL flats are inundated during high tide and exposed during low tide [1]. As the boundary area between marine and terrestrial environments, tidal flats can provide enormous ecosystem services, such as supporting abundant biodiversity, absorbing many pollutants, and storing blue carbon [2]. More-

Manuscript received November 22, 2015; revised April 25, 2016, July 11, 2016, August 9, 2016, and September 19, 2016; accepted October 2, 2016. Date of current version November 9, 2016. This work was supported from the Strategic Priority Research Program of the Chinese Academy of Sciences (XDA11020000), the Key Research Program of the Chinese Academy of Sciences (KZZD-EW-14), the Basic Special Program of Ministry of Science and Technology (2014FY210600), and the Talent Fund of Yantai Institute of Coastal Zone Research, Science, and Technology Project of Yantai (2014ZH085). (Corresponding author: Zhiqiang Gao.)

X. Liu, J. Ning, X. Yu, and Y. Zhang are with the Key Laboratory of Coastal Environmental Processes and Ecological Remediation, Yantai Institute of Coastal Zone Research, Chinese Academy of Sciences, Yantai 264003, China (e-mail: xyliu_gis@yeah.net; jcning@yic.ac.cn; yuxiangyt@126.com; zhangyuanyuan_gis@126.com).

Z. Gao is with the Key Laboratory of Coastal Environmental Processes and Ecological Remediation, Yantai Institute of Coastal Zone Research, Chinese Academy of Sciences, Yantai 264003, China (e-mail: zqgao@yic.ac.cn).

Color versions of one or more of the figures in this paper are available online at http://ieeexplore.ieee.org.

Digital Object Identifier 10.1109/JSTARS.2016.2616514 over, tidal flats can be developed for agricultural, industrial, and tourism uses and provide the foundation for regional development [3]. However, massive reclamation activity, flux in sedimentation, and sea level rise [4] have contributed to the rapid changing of tidal flats, and accurate knowledge of their status and spatial extent is urgently required.

Due to slope gradients, tides, waves, and other sedimentary factors [5], the inundation frequencies of different locations within tidal flats are remarkably different. Accordingly, tidal flats can be divided into three zones: the supratidal zone, which is located above mean high-water spring tides and is intermittently inundated; the intertidal zone, which lies between mean high-water spring tides and mean low-water spring tides and is most regularly inundated and exposed to air; and the subtidal zone, which occurs below mean low-water spring tides and is rarely exposed to air [1]. Different frequencies lead to different but important ecological characteristics and functions of these three zones. The supratidal zone is particularly important for tourism, fishery, and other human activities, and its changes cause hydrodynamic boundary conditions changes during storm surges [6]. The intertidal zone is the most productive ecosystem on earth and can provide safe spawning areas and nurseries for countless species of fish and crustaceans [7]. The subtidal zone is also a habitat for a large number of plants and animals, and its change can effectively reflect the erosion or accretion of tidal flats [8].

It is very difficult to estimate tidal flats across a large area using field observations because tidal flats are hard to access and are only exposed for short periods. Remote sensing, which can observe a large area repeatedly, is an effective tool for overcoming these difficulties and monitoring tidal flats [9]. Previous studies have used multiple boundaries between a water body and an exposed land mass in a remotely sensed image, known as waterlines [10], to identify tidal flats. As the tide constantly moves in a horizontal direction, the waterline represents information on the location of the tide when the satellite transits. Approaches to extract the extent of tidal flats using waterlines fall mostly within two categories: 1) waterlines are calculated from imagery taken as close as possible to the estimated time of high or low tide based on tide models [11] or tide gauge data [12], [13], and 2) the outermost waterline is selected from a series of waterlines extracted from multitemporal remote-sensing images [14]-[16]. For the first category, methods only apply waterlines at one moment to delineate tidal flats. Aside from the dynamic height of the tides, tides in the same area can also vary day to day based on weather or landform variables. As 


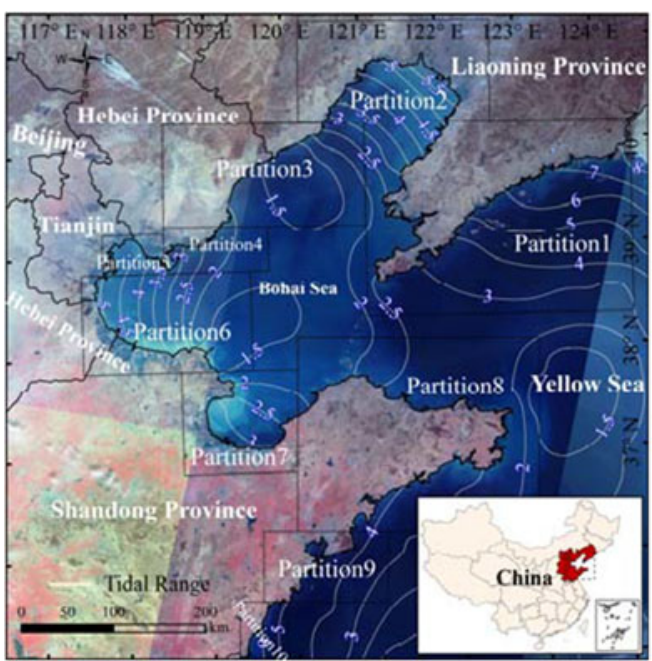

Fig. 1. HJ-1A images of the Bohai Rim with a location map. Ten Partitions of the study area.

a result, these methods do not capture the dynamic nature of tidal fluctuations, and the extent of tidal flat estimates can be highly imprecise. Although the second method uses multitemporal remote-sensing images, they have limited precision due to the resolution of the available data. For example, high-spatial resolution imagery, such as SPOT [14] and the Landsat Archive (MSS, TM, ETM+, or Landsat8) [15], [16], have a low temporal resolution, while images with a higher temporal resolution, such as those derived from the MODIS, have a coarse spatial resolution $(250 \mathrm{~m}$ ) [16]. More importantly, these methods can only extract the extent of tidal flats but cannot determine the three functionally different zones within tidal flats. To understand and use tidal flats better, it is necessary to determine the extent of the three zones.

Here, we present the utility of using higher resolution imagery in calculating multiple waterlines to estimate the total extent of tidal flats and their segmentation into functionally different zones. We used Chinese HJ-1A/B satellite images with a high temporal resolution of two days and a high spatial resolution of $30 \mathrm{~m}$ to generate a larger set of waterlines than would be possible with other images. Then, by analyzing waterlines at a small spatial scale with Digital Shoreline Analysis System (DSAS) [17] software and determining the mean high- and lowtide waterlines with Jenks Natural Breaks [18], we divided tidal flats into supratidal zones, intertidal zones, and subtidal zones. We demonstrated the relevance of this method for mapping tidal flats in the Bohai Rim, China, a region with extensive tidal flats and a changing coastal environment.

\section{RESEARCH METHODS}

\section{A. Study Area}

The Bohai Rim comprises the entire coastal area of the Bohai Sea and a portion of the coastal area of the Yellow Sea (see Fig. 1), which includes the five provinces of Beijing, Tianjin, Hebei, Liaoning, and Shandong and covers approximately 52.34 million ha. Coastlines in this region are very rich and long, $5837 \mathrm{~km}$ [19], and include many extensive tidal flats, including the following: Liaodong Bay, Bohai Bay, Yellow River Delta, Laizhou Bay, and Jiaozhou Bay. These coastal wetlands are critical foraging habitats where shorebirds along the East Asian-Australasian Flyway refuel during both northward and southward migrations [7]. Due to rapid economic and population growth, tidal flats in the Bohai Rim have experienced a huge change as they have been lost to development [7], [8]. This loss of tidal flats has resulted in severe environmental problems, leading to a dramatic reduction of biodiversity and a significant decrease in coastal ecosystem services. To promote a sustainable development in coastal areas, there is an urgent need to understand the distribution and status of tidal flats in the Bohai Rim.

\section{B. Data Selection and Preprocessing}

To maximize the accurate extraction of the extent of tidal flats, two selection criteria were important to choose a suitable satellite-based imagery. First, the temporal resolution needed to be high enough to ensure that the images selected were associated with the greatest extent of different tidal conditions in a small time interval (i.e., $<1$ year), which can reduce short-term variation in the tidal flat extent. Second, the spatial resolution needed to be sufficient for mapping tidal flats within a narrow width.

In this study, we used Chinese-designed HJ-1A/B satellite imagery, which has both a high temporal resolution and high spatial resolution, as the main data source. HJ-1A/B satellites were recently launched by China. Each satellite carries two CCD sensors side-by-side, and each sensor has three visible bands and one near-infrared band [20]. All of the 1794 images available from the HJ-1A/B in our study area in 2014 were downloaded from the China Center for Resources Satellite Data and Application (CRESDA, http://www.cresda.com). Eighteen Landsat8 images covering the study area were downloaded from United States Geological Survey (USGS, http://glovis.usgs.gov/) as a reference for geometric corrections.

Batch band composition of images was conducted using the Python programming language, while a third-order polynomial was used in ArcGIS software for geometric corrections. The total root mean square (RMS) error during geometric correction was controlled within one pixel for each image. Finally, mosaics of images with the same acquisition time were created using ENVI+IDL. To increase the number of available images, the study area was divided into ten small areas based on the distribution characteristics of tidal flats [12] (see Fig. 1). Mosaics of images were clipped into each small area and loaded into the ArcGIS software. Images with better quality (cloudfree and with clear waterlines) were identified visually. Each area involved at least 65 available images, and the details on the number of selected images are listed in Table I.

\section{Waterline Extraction}

Currently, many methods have been developed to delineate the waterline, including manual digitization [14], [15], edge detection [21], and threshold segmentation [22], [23]. Manual 
digitization is a straightforward and effective procedure that can assure reliability and accuracy [14], but it is very time consuming. Edge detection uses detection operators to track the boundary of water and is mainly suitable for images with a high contrast. Threshold segmentation can separate water from other land-cover types effectively despite impurities (nonwater information) in the water. However, tidal conditions, remnant surface water, and other environmental parameters often result in imprecise or "fuzzy" waterline locations [24]. We adopted a semiautomatic method in this study to extract waterlines that minimized this imprecision.

Generally, tidal flats are submerged during incoming tides and have clear waterlines, while a large quantity of remnant surface water leads to blurry waterlines during falling tides. Therefore, available remote-sensing images were classified as either "clear" or "fuzzy" using visual interpretation. For images classified as “clear," we applied "Canny edge detection" to extract waterlines. For images classified as "fuzzy," we used a combination of the NIR and a visible band to reduce the effect of turbidity [24]. NDWI, the normalized difference water index proposed by S.K. McFeeters, is a high-performing and widely accepted method for delineating open water features [25] that has been applied successfully to extract waterlines in highly turbid coastal area (e.g., typical coastal wetlands of Bohai Rim [23], tidal flats along the Jiangsu middle coast in China [22], and tidal flats across East Asia [11]). Therefore, this study used NDWI to extract waterlines from "fuzzy" waterlines. NDWI's formula is as follows:

$$
\text { NDWI }=\frac{\text { Green }- \text { NIR }}{\text { Green }+ \text { NIR }}
$$

where Green and NIR represent the green light band and near-infrared band, respectively, corresponding to the second and fourth bands of HJ-1A/B satellite imagery. A threshold value was selected for NDWI images to generate binary images (land/water). After automatic vectorization, waterlines with "fuzzy" locations could be delineated. Finally, each waterline that was extracted automatically was checked by visual interpretation and, where needed, was corrected by modifying inaccurate waterlines and linking discontinuous waterlines.

\section{Tidal Flat Delineation and Zones}

To map tidal flats, we selected the outermost boundaries of all waterlines as the total extent of tidal flats [10], [14]-[16]. This process consisted of merging multiple waterlines and transformations from lines to polygons, which were implemented in ArcGIS 10.0 software. Tidal flats can be divided into three zones (the supratidal, the intertidal, and the subtidal) and are demarcated as mean high water and mean low water during spring tides [1]. Spring tides, which are rare events, occur twice a month, around the full moon and the new moon. Therefore, it is very difficult to ensure that available images capture all high- and low-water spring tides. To determine different zones of tidal flats, we took the mean high tides and mean low tides as dividing lines. Thus, we defined the supratidal zone as the area above the mean high-tide waterlines, the intertidal zone as the area between the mean high-tide and mean low-tide water-
TABLE I

NUMBER OF AVAILABLE IMAGES AND SUPRATIDAL-TO-SUBTIDAL TRANSECTS

\begin{tabular}{lccccccccccc}
\hline \hline Partition & 1 & 2 & 3 & 4 & 5 & 6 & 7 & 8 & 9 & 10 & Total \\
\hline $\begin{array}{l}\text { Available Image } \\
\text { Transect }\end{array}$ & 74 & 72 & 68 & 68 & 76 & 82 & 90 & 65 & 77 & 78 & 75 \\
\hline \hline
\end{tabular}

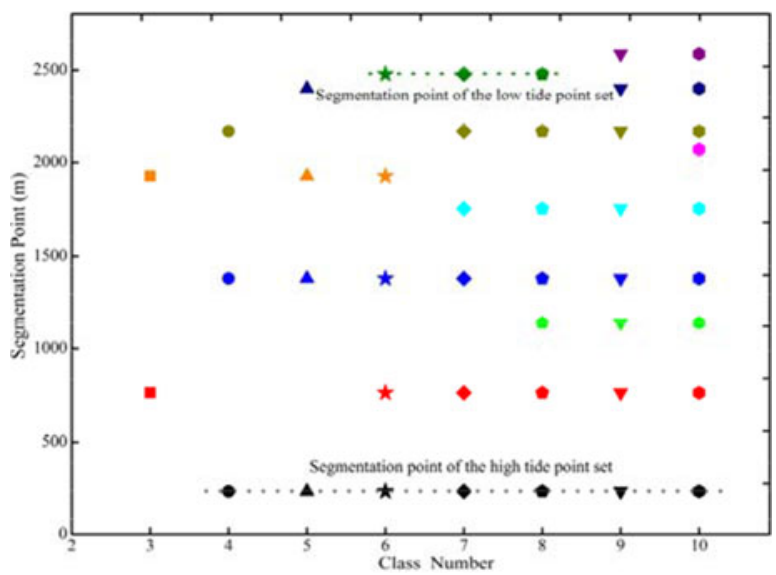

Fig. 2. Relation between the segmentation point and class number.

lines, and the subtidal zone as the area below the mean low-tide waterlines.

Due to the variation in daily tide heights and the influences of weather and topography, tidal movements in the same area can vary. As a result, waterlines at different times may cross each other. To identify these discrepancies, tidal flats should be analyzed at a small spatial scale. A transect was generated every $100 \mathrm{~m}$ along the coast [26] of the study area by using DSAS software, and the total of transect was 17099 (see Table I). Each transect and each waterline had an intersection point, which represented the estimated location (distance from shore) of the tide at each satellite transit time.

Each transect had at least 65 tidal points that were estimated at different times and under different tidal conditions. The distances between each tidal point and the coast were calculated. Then, the tidal points were classified according to distances. This study defined the class that was closest to the coast as the high-tide point set, the class that was furthest from the coast as the low-tide point set, and other classes as middle-tide point sets. The averages of the high- and low-tide point sets were calculated to obtain the mean high-tide and mean low-tide point on each transect. The mean high-tide and mean low-tide points on all transects were linked to obtain the mean high-tide and mean low-tide waterlines, which were used for dividing tidal flats.

To define high, middle, and low classes, we applied the Jenks Natural Breaks [18]. This classification method is a form of data clustering that seeks natural "faults" and "discontinuity points" according to intrinsic statistical characteristics of data. This method maximizes variance between classes and minimizes variance within classes to achieve the best classification.

To determine the best segmentations of the high-tide and the low-tide point sets, we first analyzed the data characteristics of tidal points. As shown in Fig. 2, we found that regardless of 


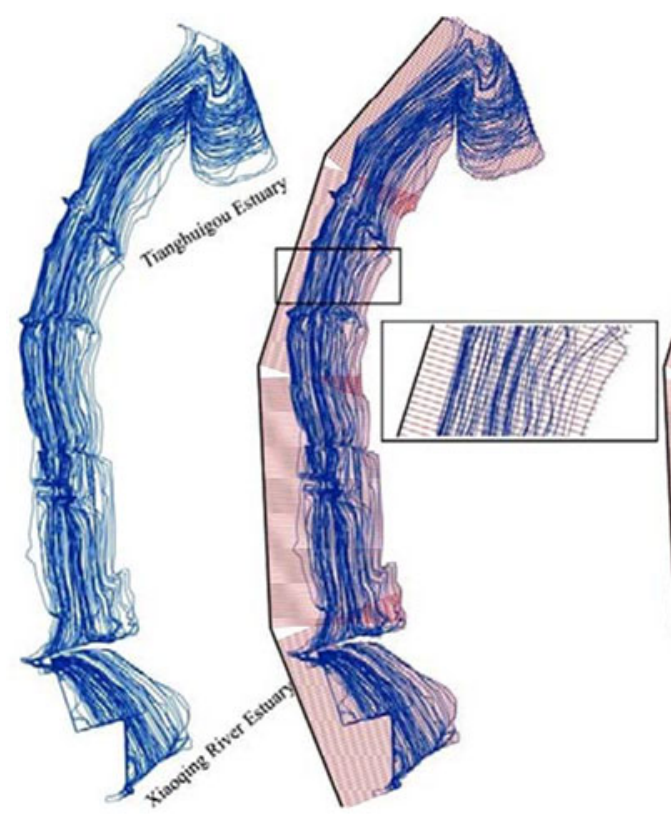

(a) (b)

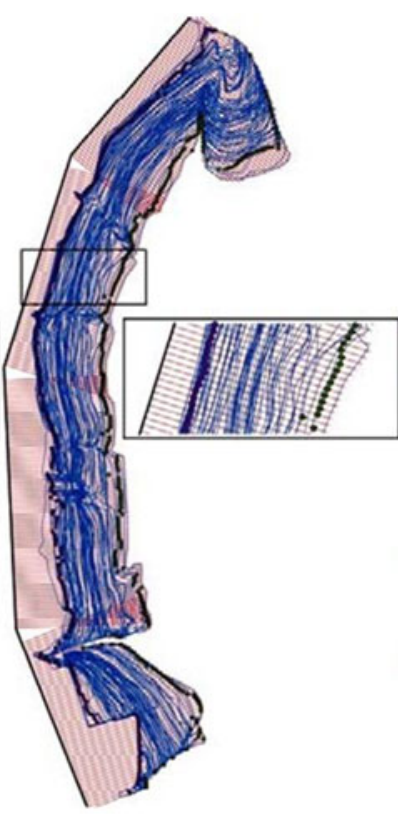

(c)

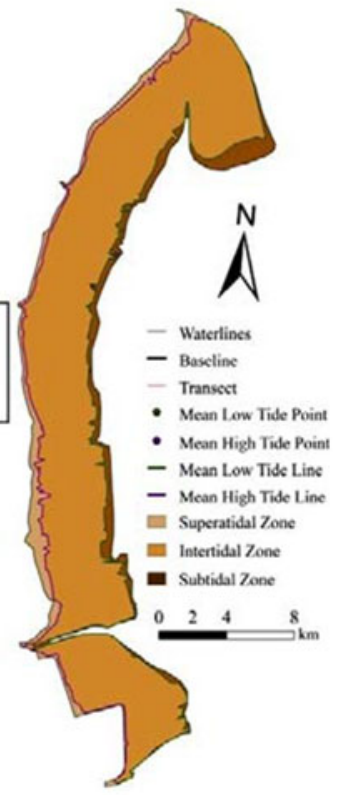

(d)

Fig. 3. Process of tidal flat delineation and zoning.

the number of classes, the best segmentations of high- and lowtide point sets were similar. Therefore, distances of tidal points along each transect were classified into three, four, five, and ten classes. Classifications of more than ten classes removed the clustering characteristics of the data. Then, the modes of the segmentation points of the class that was closest to the coast and the class that was farthest from the coast were selected as segmentation points of the high-tide point set and the low-tide point set of every transect. After that, the mean high-tide and mean low-tide waterlines were determined. Finally, we calculated three different zones of tidal flats. This process is explained in detail for a section from the Tianshuigou estuary to the $\mathrm{Xi}$ aoqing River estuary in the Yellow River Delta (see Fig. 3). In this section, 90 waterlines were estimated at different times in 2014 [see Fig. 3(a)]. DSAS software was then used to generate 498 transects, which were spaced $100 \mathrm{~m}$ along the coast, with 90 tide points on each transect [see Fig. 3(b)]. Jenks Natural Breaks classification then allowed us to identify the set of tidal points to calculate the mean high-tide and low-tide points [see Fig. 3(c)]. Finally, the outermost boundaries of 90 waterlines were extracted to obtain the full extent of tidal flats and all of the mean high-tide and low-tide points were linked to determine the three zones of tidal flats [see Fig. 3(d)].

\section{E. Accuracy Assessment}

To validate the accuracy of the proposed method, the positional accuracy of waterlines, the basis of mapping tidal flats, must be analyzed. Because a waterline does not have a definite position, we used a buffering method [27] to estimate its positional accuracy. A buffer with a certain width around the assessed waterline was created, called a "water stripe." The wider the "water stripe," the easier it is to correctly separate land from water. Under a certain buffer width, if some parts of the "water stripe" could separate land from water correctly, the length of waterline segments within them was calculated. We introduced the separation degree to illustrate the positional accuracy of the waterline. The separation degree $(S)$ is formulated as follows:

$$
S(x)=\frac{L_{1}}{L_{2}}
$$

where $x$ is the buffer width of the "water stripe," $S(x)$ is the separation degree of $x, \mathrm{~L}_{1}$ is the length of waterline segments within separating correctly water stripe parts, and $\mathrm{L}_{2}$ is the total length of the whole waterline. Initially, we randomly selected 20 waterlines of clear images and fuzzy images. Then, we generated three buffers with $30 \mathrm{~m}$ (one pixel), $60 \mathrm{~m}$ (two pixels), and $90 \mathrm{~m}$ (three pixels) for every waterline. By computing $S(30)$, $S(60)$, and $S(90)$ using an independent analyst with a rigorous visual interpretation, the positional accuracy of every waterline could be estimated.

To assess the accuracy of the extents of the estimated tidal flat, this study adopted a widely used accuracy assessment method termed a confusion matrix [28]. First, we generated a 5-km buffer around tidal flats as an assessment area. This area, where the proportion of tidal flats and other land types was not too imbalanced, contained the actual tidal flats. Second, we generated 240 sample points using a stratified random sampling within the assessment area for the two output classes (tidal flats and other). Finally, we extracted all of the sample points from the tidal flat map as map data. Reference data were derived by an independent analyst who classified each sample point as either a tidal flat or other using all of the available bands of the low-tide $\mathrm{HJ}-1 \mathrm{~A} / \mathrm{B}$ satellite images as well as other available information (such as field investigation data and Google Earth) [11]. It should be 
TABLE II

ACCURACY ASSESSMENT FOR EXTRACTING WATERLINES

\begin{tabular}{lccccc}
\hline \hline Type & $\begin{array}{c}\text { Postprocessing } \\
\text { Step }\end{array}$ & $\begin{array}{c}\text { Waterline } \\
\text { Length }(\mathrm{km})\end{array}$ & $\begin{array}{c}S(30) \\
(\%)\end{array}$ & $\begin{array}{c}S(60) \\
(\%)\end{array}$ & $\begin{array}{c}S(90) \\
(\%)\end{array}$ \\
\hline Clear Images & Before & 13264 & 66.7 & 76.3 & 85.7 \\
& After & 12669 & 90.6 & 96.1 & 98.7 \\
Fuzzy Images & Before & 13181 & 54.3 & 64.7 & 73.1 \\
& After & 10798 & 68.5 & 85.5 & 95.1 \\
All Images & Before & 26446 & 60.5 & 70.5 & 79.4 \\
& After & 23467 & 80.4 & 91.2 & 97.0 \\
\hline \hline
\end{tabular}

TABLE III

CONFUSION MATRIX FOR MAPPING TIDAL FLATS

\begin{tabular}{|c|c|c|c|c|c|}
\hline & & \multicolumn{2}{|c|}{ Classified } & \multirow[t]{2}{*}{$\Sigma$} & \multirow[t]{2}{*}{ Producer's Accuracy (\%) } \\
\hline & & Tidal Flat & Other & & \\
\hline \multirow[t]{3}{*}{ Reference } & Tidal flat & 111 & 6 & 117 & 94.9 \\
\hline & Other & 9 & 114 & 123 & 92.7 \\
\hline & $\Sigma$ & 120 & 120 & 240 & \\
\hline \multicolumn{2}{|c|}{ User's accuracy (\%) } & 92.5 & 95.0 & & \\
\hline \multicolumn{2}{|c|}{ Overall accuracy (\%) } & & & & 93.8 \\
\hline
\end{tabular}

Notes: The matrix shows the actual and classified cases in the rows and columns, respectively. The proportion of correctly allocated cases indicates the overall classification accuracy [28].

noted that there is a time span between $\mathrm{HJ}-1 \mathrm{~A} / \mathrm{B}$ satellite imagery and Google Earth data. Therefore, human experience and judgments could be important for satisfactory reference data [29]. We computed the confusion matrix of the resulting map with respect the reference expert data and estimated accuracy measures that include overall, user's and producer's accuracy.

\section{RESULTS}

As summarized in Table II, using the semiautomatic extraction method in our paper could ensure that $91.2 \%$ of waterlines, both on clear images and fuzzy images, have an extracted accuracy within two pixels. With the help of manual corrections, $90.6 \%$ of waterlines on clear images had a positional accuracy within $30 \mathrm{~m}, 96.1 \%$ of waterlines' accuracy was within $60 \mathrm{~m}$, and $98.7 \%$ of waterlines' accuracy was within $90 \mathrm{~m}$. This high precision indicates that "Canny edge detection" can extract waterlines on clear images and result in a positional accuracy of the vast majority of waterlines within one pixel. For fuzzy images, the proportions of waterlines' accuracy within 30,60 , and $90 \mathrm{~m}$ were $68.5 \%, 85.5 \%$, and $95.1 \%$, respectively. While using a combination of the NIR and a visible band that is not always reliable when turbidity is extremely high [24], we successfully used the NDWI combined with visual interpretation to extract waterlines on fuzzy images and ensure that most waterlines' accuracies fall within three pixels. However, further evaluation of waterline mapping on tidal flats is required, preferably with on-ground reference data collected at known tide stages and heights that can be matched to the time imagery taken [24].

As shown in Table III, the overall classification accuracy was 93.8\% for mapping tidal flats, with a user's accuracy of $92.5 \%$ and a producer's accuracy of $94.9 \%$. The reasons for such high
TABLE IV

Tidal Flat AREA AND ZoNES IN 2014

\begin{tabular}{|c|c|c|c|c|c|c|c|}
\hline \multirow[t]{2}{*}{ Partition } & \multirow[t]{2}{*}{ Tidal Flat Area $\left(\mathrm{km}^{2}\right)$} & \multicolumn{2}{|c|}{ Supratidal Zone } & \multicolumn{2}{|c|}{ Intertidal Zone } & \multicolumn{2}{|c|}{ Subtidal Zone } \\
\hline & & $\begin{array}{l}\text { Area } \\
\left(\mathrm{km}^{2}\right)\end{array}$ & $\begin{array}{l}\text { Proportion } \\
\quad(\%)\end{array}$ & $\begin{array}{l}\text { Area } \\
\left(\mathrm{km}^{2}\right)\end{array}$ & $\begin{array}{l}\text { Proportion } \\
\text { (\%) }\end{array}$ & $\begin{array}{l}\text { Area } \\
\left(\mathrm{km}^{2}\right)\end{array}$ & $\begin{array}{l}\text { Proportio } \\
\qquad \%)\end{array}$ \\
\hline 1 & 607 & 26 & 4.3 & 510 & 84.0 & 71 & 11.7 \\
\hline 2 & 631 & 27 & 4.3 & 520 & 82.4 & 84 & 13.3 \\
\hline 3 & 39 & 2 & 5.1 & 35 & 89.7 & 2 & 5.1 \\
\hline 4 & 106 & 6 & 5.7 & 93 & 87.7 & 7 & 6.6 \\
\hline 5 & 131 & 7 & 5.3 & 115 & 87.8 & 8 & 6.1 \\
\hline 6 & 455 & 19 & 4.2 & 376 & 82.6 & 62 & 13.6 \\
\hline 7 & 681 & 48 & 7.0 & 578 & 84.9 & 55 & 8.1 \\
\hline 8 & 256 & 11 & 4.3 & 224 & 87.5 & 21 & 8.2 \\
\hline 9 & 137 & 8 & 5.8 & 113 & 82.5 & 16 & 11.7 \\
\hline 10 & 49 & 2 & 4.1 & 43 & 87.8 & 4 & 8.2 \\
\hline Total & 3093 & 156 & 5.0 & 2607 & 84.3 & 330 & 10.7 \\
\hline
\end{tabular}

levels of accuracy include the following: 1) only two mapping classifications exist in the accuracy assessment [28]; 2) the high resolution of the available imagery allowed for the estimation of many waterlines at fine spatial scales; 3 ) all of the waterlines that were extracted automatically were manually corrected to ensure accuracy [15], [22]; and 4) HJ-1A/B images were used for validation [11]. Misclassified sample points were mainly located in the landward region of tidal flats. Due to the limited number of available images during a spring high tide, a relatively rare event, the vast majority of misclassified points occurred on slivers between the natural coastline or the seawall and mean high-tide waterline that we obtained [11]. Moreover, coastal areas under active development and ships docked in harbor were also sometimes mistaken for tidal flats. However, such misclassifications were removed manually during the postprocessing step when identified.

In 2014, the estimated extent of tidal flats in Bohai Rim, China, was $3093 \mathrm{~km}^{2}$, among which the area of supratidal, intertidal, and subtidal zones were 156,2607 , and $330 \mathrm{~km}^{2}$, respectively, and they accounted for 5.0\%, 84.3\%, and $10.7 \%$ of the tidal extent, respectively (see Table IV). Compared with the actual tidal flats, the result derived from our method was conservative. Although we gathered many images, it was difficult to ensure that the available imagery included tidal extremes. Moreover, the mean high tides are closer to the sea than the mean high-water spring tides, and the mean low tides are closer to the coast than the mean low-water spring tides. Therefore, the intertidal zones in this study were also smaller than the actual intertidal zones. Nevertheless, the accuracy of the method in this study for mapping tidal flats could meet widely accepted targets for remote sensing [28].

\section{DISCUSSION}

The formation and development of tidal flats are related to the area's slope: regions with steep slopes have weak sedimentation, and tidal flats in them have a narrow width [12]. The closer the distance to the coast, the steeper the slope [1]. For this reason, the extent of the supratidal zone measured in this study is small. On the other hand, enormous coastal engineering relieves the 
sharp contradiction between the land and people in the study area, which leads to the loss of the supratidal zone [8]. For example, salt fields and aquaculture have reduced the supratidal zone in Caofeidian of $191.8 \mathrm{~km}^{2}$ from 1981 to 2011 [13], and port-vicinity industry and tourism have resulted in an annual sea reclamation area of $96.96 \mathrm{~km}^{2}$ along the northwest coast of Bohai Bay from 2004 to 2010, 36.4\% of which is coastal tidal flat reclamation [30]. Interestingly, the supratidal zones of the Yellow River Delta (partition 7), a region with a gentle slope that has been little influenced by human activities [26], are the largest, illustrating that slope and coastal engineering are the two reasons for the small area of the supratidal zone. Moreover, the tidal range is also a crucial factor for the formation and development of tidal flats. When sediment is fully supplied, the width of the tidal flats is adjusted to the size of the tidal range [12]. Therefore, there is a positive correlation relationship between the tidal range and the area of the subtidal zones. From Fig. 1 and Table IV, it is evident that regions where the tidal range is long (Partitions 1, 2, 6, and 9) also have large subtidal zones.

To guarantee the quality of the data used for analysis, this study used Chinese-designed HJ-1A/B satellite images with a high temporal resolution (2 d) and a high spatial resolution (30 m). By dividing the Bohai Rim into ten small areas, many images with a poor overall quality could still be used, as some sections of these images were of sufficient quality. The high temporal resolution of these images allowed us to identify more than 65 images that could be used in each of these ten areas (mean $=75$, $\max =90$ ). In turn, the high number of images allowed us to estimate a full set of waterlines across a greater range of tide heights within one year compared to previous studies. Then, we extracted the extent of available tidal flats at a much greater precision than had been previously achieved.

Current studies for mapping tidal flats, both when analyzing waterlines related to the low (high) tide time [8], [11]-[13] and when choosing the outmost boundary of multitemporal waterlines [10], [15]-[17], do not identify the supratidal zone, the intertidal zone and the subtidal zone, as was successfully done here. We used DSAS software and Jenks Natural Breaks to achieve zoning of tidal flats, which is meaningful for government planners, ecologists and geographers. For example, the reclamation plans of the Bohai Rim are $1087 \mathrm{~km}^{2}$ by 2020 according to the master plan for coastal provinces [7]. Our results are especially relevant for the supratidal zone and could provide some reference for government decisions. The tidal flats in the study area were located in the East Asian-Australasian Flyway and are very important for many waterbirds, such as the red knot, the great knot, and the bar-tailed. The area, distribution, and relationship with waterbird population decline in intertidal zones have been attracting ecologists' attention [5]. There are more than 100 rivers emptying into the sea in the Bohai Rim, including the Yellow, Liaohe, Hai he, and Luanhe Rivers, as well as other famous rivers, and the sediments carried by them provide a rich source for tidal flat deposits [23]. Determining the extent of the subtidal zone in our study helps us to effectively analyze tidal flat evolution. Moreover, this improvement in mapping will prompt a more comprehensive understanding of the full extent of tidal flats, something that is required if a sufficient area of these unique ecosystems is to be maintained.

The ecosystem value of tidal flats mainly relate to benthic macrofauna, as they decompose the organic matter, recycle nutrients, and are a crucial food source to many other species, including human beings. Because it determines the duration of the feeding time, exposure time may be a restrictive factor for some benthic macrofauna, such as Cerastoderma edule, Mytilus edulis, and Meloidogyne arenaria, and is thus an important predictor of benthic macrofauna distributions [31]. Our method could generate a sufficient number of waterlines that could then be classified using Jenks Natural Breaks or other classification methods, (e.g., equal interval and quantile) to divide tidal flats into some zones that have different exposure times and would likely contain the richest benthic macrofauna communities. This implication would be fundamental to describe the assemblage composition of benthic macrofauna, understanding tidal flat ecosystems, and even determining the influence of anthropogenic effects.

\section{CONCLUSION}

Tidal flats are rapidly disappearing, and an accurate understanding of their status and the zones within them is urgently required. This study highlights the advantages of using higher resolution imagery, such as that available from the Chinese HJ1A/B satellites, to generate more precise mapping of tidal flats. We used a semiautomatic method to extract a series of remotely sensed waterlines and ensure their positional accuracy within two pixels. By estimating over 65 waterlines within one year, we were able to more fully capture the full range of tide heights, enabling an improved boundary around tidal flats. More importantly, by clustering waterlines, we can also identify three distinct zones of inundation frequency within tidal flats. The spatial understanding of supratidal zones, intertidal zones and subtidal zones should aid in sustainable development planning of coastal areas. In the Bohai Rim, China, in 2014, the extent of tidal flats was $3093 \mathrm{~km}^{2}$, among which the area of supratidal, intertidal, and subtidal zones accounted for $5.0 \%, 84.3 \%$, and $10.7 \%$, respectively. In this study, the accuracy assessment showed an overall classification accuracy of $93.8 \%$, with a user's accuracy of $92.5 \%$ and a producer's accuracy of $94.9 \%$.

This method could provide a useful tool for studies on tidal flats of East Asia and be applied to other sensor data. In future studies, we will further address waterline extraction approaches to improve our method and implement this method to understand morphological change in tidal flats, particularly with regard to ecosystem services and human impacts.

\section{ACKNOWLEDGMENT}

The authors would like to thank the Center for Resources Satellite Date and Application (CRESDA, China) and the United States Geological Survey (USGS, USA) for providing the satellite images. They would also like to thank four anonymous reviewers and editors for their detailed and constructive comments on this paper. 


\section{REFERENCES}

[1] C. Li, P. Wang, and D. Fan, "Tidal flats, open ocean coasts," in Encyclopedia of Coastal Science. Springer, 2005, pp. 975-978.

[2] Z. Ma et al., "Rethinking China's new great wall," Science, vol. 346, pp. 912-914, 2014.

[3] H. K. Lotze et al., "Depletion, degradation, and recovery potential of estuaries and coastal seas," Science, vol. 312, pp. 1806-1809, 2006.

[4] H. Wang et al., "Recent changes in sediment delivery by the Huanghe (Yellow River) to the sea: Causes and environmental implications in its estuary," J. Hydrol., vol. 391, pp. 302-313, 2010.

[5] N. Baghdadi, N. Gratiot, J.-P. Lefebvre, C. Oliveros, and A. Bourguignon, "Coastline and mudbank monitoring in French Guiana: Contributions of radar and optical satellite imagery," Can. J. Remote Sensing, vol. 30, pp. 109-122, 2004.

[6] B. S. Halpern et al., "A global map of human impact on marine ecosystems," Science, vol. 319, pp. 948-952, 2008.

[7] J. MacKinnon, Y. I. Verkuil, and N. Murray, "IUCN situation analysis on East and Southeast Asian intertidal habitats, with particular reference to the Yellow Sea (including the Bohai Sea)," Occasional Paper of the IUCN Species Survival Commission, vol. 47, 2012.

[8] N. J. Murray, R. S. Clemens, S. R. Phinn, H. P. Possingham, and R. A. Fuller, "Tracking the rapid loss of tidal wetlands in the Yellow Sea," Frontiers Ecol. Environ., vol. 12, pp. 267-272, 2014.

[9] S. R. Phinn, 1. Menges, G. J. Hill, and M. Stanford, "Optimizing remotely sensed solutions for monitoring, modeling, and managing coastal environments," Remote Sensing Environ., vol. 73, pp. 117-132, 2000.

[10] D. Mason, I. Davenport, G. Robinson, R. Flather, and B. McCartney, "Construction of an inter-tidal digital elevation model by the 'water-line' method," Geophys. Res. Lett., vol. 22, pp. 3187-3190, 1995.

[11] N. J. Murray, S. R. Phinn, R. S. Clemens, C. M. Roelfsema, and R. A. Fuller, "Continental scale mapping of tidal flats across East Asia using the Landsat archive," Remote Sensing, vol. 4, pp. 3417-3426, 2012.

[12] Q. Han, "Research on the status of tidal resources in China using remote sensing technology," M.S. thesis, Dept. Geographic. Sci., Nanjing Normal Univ., Nanjing, China, 2011.

[13] X. D. Wan, C. Fang, H. Kang, H. L. Xie, F. T. Liu, and L. Meng, "Remote sensing monitoring of the Caofeidian tidal zone evolution," Marine Sci. Bull., vol. 33, pp. 559-565, 2014.

[14] L. Chen, "Detection of shoreline changes for tideland areas using multitemporal satellite images," Int. J. Remote Sensing, vol. 19, pp. 3383-3397, 1998.

[15] B. Zhao, H. Q. Guo, Y. Yan, Q. Wang, and B. Li, "A simple waterline approach for tidelands using multi-temporal satellite images: A case study in the Yangtze Delta," Estuarine, Coastal Shelf Sci., vol. 77, pp. 134-142, 2008.

[16] Y. X. Liu, M. C. Li, M. X. Zhou, K. Yang, and L. Mao, "Quantitative analysis of the waterline method for topographical mapping of tidal flats: A case study in the Dongsha Sandbank, China," Remote Sensing, vol. 5, pp. 6138-6158, 2013.

[17] E. R. Thieler, E. A. Himmelstoss, J. L. Zichichi, and A. Ergul, "The Digital Shoreline Analysis System (DSAS) Version 4.0-An ArcGIS Extension for Calculating Shoreline Change," U.S. Geological Survey, Woods Hole, MA, Open-File Rep. 2008-1278, 2009.

[18] G. F. Jenks, "The data model concept in statistical mapping," Int. Yearbook Cartogr, vol. 7, pp. 186-190, 1967.

[19] N. Xu, Z. Gao, and J. Ning, "Analysis of the characteristics and causes of coastline variation in the Bohai Rim (1980-2010)," Environ. Earth Sci., vol. 75, pp. 1-11, 2016.

[20] M. M. Jia, Z. M. Wang, B. Zhang, and Z. Ding, "Land cover classification of compositing HJ-1 and Modis data based on object-based method," Geomatics Inform. Sci. Wuhan Univ., vol. 39, pp. 305-310, 2014.

[21] K. White and H. M. El Asmar, "Monitoring changing position of coastlines using thematic mapper imagery, an example from the Nile Delta," Geomorphology, vol. 29, pp. 93-105, 1999.

[22] Y. X. Liu, M. C. Li, L. Mao, L. Cheng, and K. F. Chen, "Seasonal pattern of tidal-flat topography along the Jiangsu Middle Coast, China, using HJ-1 optical images," Wetlands, vol. 33, pp. 871-886, 2013.

[23] Y. D. Wang, X. Y. Hou, P. Shi, and L. J. Yu, "Detecting shoreline changes in typical coastal wetlands of Bohai Rim in North China," Wetlands, vol. 33, pp. 617-629, 2013.

[24] J. H. Ryu, J. S. Won, and K. D. Min, "Waterline extraction from Landsat TM data in a tidal flat: A case study in Gomso Bay, Korea," Remote Sensing Environ., vol. 83, pp. 442-456, 2002.

[25] S. McFeeters, "The use of the Normalized Difference Water Index (NDWI) in the delineation of open water features," Int. J. Remote Sensing, vol. 17, pp. 1425-1432, 1996.
[26] Y. Liu, H. Huang, Z. Qiu, and J. Fan, "Detecting coastline change from satellite images based on beach slope estimation in a tidal flat," Int. $J$. Appl. Earth Observation Geoinform., vol. 23, pp. 165-176, 2013.

[27] M. F. Goodchild and G. J. Hunter, "A simple positional accuracy measure for linear features," Int. J. Geographical Inform. Sci., vol. 11, pp. 299-306, 1997.

[28] R. G. Congalton and K. Green, Assessing the Accuracy of Remotely Sensed Data: Principles and Practices. Florida, USA: CRC Press, 2008.

[29] Y. Yang et al., "Landsat 8 OLI image based terrestrial water extraction from heterogeneous backgrounds using a reflectance homogenization approach,” Remote Sensing Environ., vol. 171, pp. 14-32, 2015.

[30] G. R. Zhu and X. X. Gong, "Annual processes of land reclamation from the sea along the northwest coast of Bohai Bay during 1974 to 2010," Scientia Geographica Sinica, vol. 8, p. 016, 2012.

[31] T. J. Compton et al., "Distinctly variable mudscapes: Distribution gradients of intertidal macrofauna across the Dutch Wadden Sea," J. Sea Res. vol. 82, pp. 103-116, 2013.

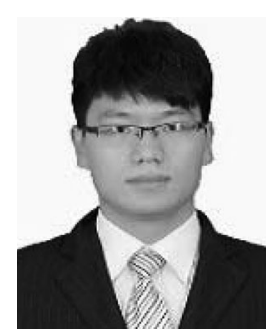

Xiangyang Liu received the M.S. degree in cartography and geographic information system from Yantai Institute of Coastal Zone Research, Chinese Academy of Sciences, Yantai, China, in 2016. He is currently working toward the Ph.D. degree at the Institute of Geographic Sciences and Natural Resources Research, Chinese Academy of Sciences, Beijing, China.

His current research interests include coastal zone remote sensing and thermal infrared quantitative remote sensing.

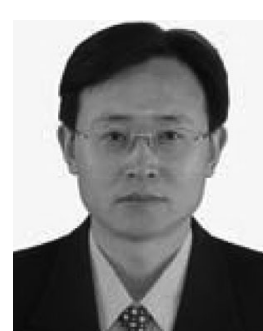

Zhiqiang Gao received the Ph.D. degree in mapping and geographic information system from the Institute of Remote Sensing Application, Chinese Academy of Sciences, Beijing, China, in 1998.

$\mathrm{He}$ is currently a Professor at the University of Chinese Academy of Sciences, Beijing, and a Research Scientist at Yantai Institute of Coastal Zone Research, Chinese Academy of Sciences, Yantai, China. His current research interests include remote sensing application and model simulation in coastal zone.

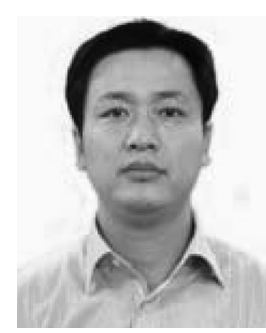

Jicai Ning received the Ph.D. degree in cartography and geographic information system from the Institute of Geographic Sciences and Natural Resources Research, Chinese Academy of Sciences, Beijing, China in 2011 .

He is currently with Yantai Institute of Coastal Zone Research, Chinese Academy of Sciences, Yantai, China. His current research interests include coastal zone digitalization and hydrological remote sensing.

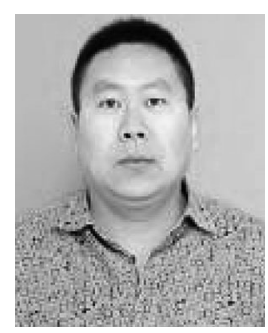

Xiang Yu received the M.S. degree in environmental science from Dalian Marine University, Dalian, China, in 2005. He is currently working toward the Ph.D. degree at Yantai Institute of Coastal Zone Research, Chinese Academy of Sciences, Yantai, China.

His current research interests include coastal zone remote sensing and quantitative remote sensing.

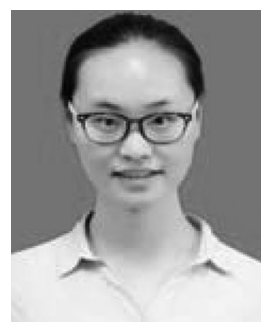

Yuanyuan Zhang received the B.S. degree in geographic information system from Shandong Normal University, Jinan, China, in 2016. She is currently working toward the M.S. degree in cartography and geographic information system at Yantai Institute of Coastal Zone Research, Chinese Academy of Sciences, Yantai, China.

Her current research interests include coastal zone remote sensing. 